\title{
Fibulin-3 and other cartilage metabolism biomarkers in relationship to calprotectin (MRP8/14) and disease activity in rheumatoid arthritis patients treated with anti-TNF therapy
}

\author{
Magdalena Kopeć-Mędrek ${ }^{A-D, F}$, Eugene J. Kucharz ${ }^{\mathrm{A}, \mathrm{E}, \mathrm{F}}$ \\ Department of Internal Medicine and Rheumatology, School of Medicine in Katowice, Medical University of Silesia, Poland \\ A - research concept and design; B - collection and/or assembly of data; C - data analysis and interpretation; \\ $D$ - writing the article; $E$ - critical revision of the article; $F$ - final approval of the article
}

Address for correspondence

Magdalena Kopeć-Mędrek

E-mail:magda.kopec@gazeta.pl

\section{Funding sources}

This study was supported by Medical University of Silesia, Katowice, Poland (KNW-1-045/N/3/0).

Conflict of interest

None declared

Received on September 28, 2016

Reviewed on October 9, 2016

Accepted on January 9, 2017

\begin{abstract}
Background. Fibulin-3 (Fib-3) is a new potential biomarker of articular cartilage metabolism.

Objectives. The aim of the study was to evaluate the effect of anti-TNF therapy on serum fibulin-3, cartilage oligomeric matrix protein (COMP), procollagen II C-propeptide (PIICP), and urinary C-terminal telopeptide of type II collagen (CTX-II) levels in relation to calprotectin (MRP8/14) and disease activity in rheumatoid arthritis patients.
\end{abstract}

Material and methods. In the study, 35 female patients with rheumatoid arthritis (RA) were investigated. The concentration of fibulin-3, COMP, PIICP, MRP8/14, and urinary CTX-II in serum was measured before and after anti-TNF therapy. Ten healthy women were investigated as the controls.

Results. The concentration of fibulin-3 in RA patients before treatment did not differ significantly from the concentration of fibulin-3 in the control group. A significantly higher concentration of fibulin-3 was noted prior to treatment in the group of women with a worse response to the therapy (non-responders) compared to the concentration of fibulin-3 in the healthy women. During the anti-TNF therapy, the serum fibulin-3 level decreased in patients. The fibulin-3 level correlated with CRP and ESR after anti-TNF treatment. Significant lowering of MRP8/14 was noted in the patients after anti-TNF therapy. No correlation between fibulin-3 and MRP8/14 was observed in the study group or in the control group.

Conclusions. During the anti-TNF therapy, the serum fibulin-3 level decreased in RA patients. Serum MRP8/14 concentration also decreased. No correlation between fibulin-3 and MRP8/14 was observed in the study group before and after the treatment. We found a poor correlation between serum fibulin-3 and other cartilage metabolism biomarkers after anti-TNF therapy.

Key words: rheumatoid arthritis, fibulin-3, calprotectin

DOI

10.17219/acem/68362

Copyright

Copyright by Author(s)

This is an article distributed under the terms of the

Creative Commons Attribution Non-Commercial License

(http://creativecommons.org/licenses/by-nc-nd/4.0/) 


\section{Introduction}

Rheumatoid arthritis (RA) is a chronic systemic inflammatory disease associated with synovial inflammation as well as cartilage and bone destruction. Production of proinflammatory cytokines: tumor necrosis factor (TNF), interleukin-6 (IL-6) and interleukin-17 (IL-17), causes the release of proteolytic enzymes, matrix metalloproteinases, and degradation of the connective tissue of the joint. Cartilage metabolism may be monitored with evaluation of the synthesis and degradation of cartilage products. Biological markers are the object of increasing interest in diagnostics and osteoarthritis (OA) monitoring. ${ }^{1}$ In the case of rheumatoid arthritis, attempts have been made for many years to find biomarkers which would facilitate monitoring the activity of the disease and predicting the response to treatment in RA patients. Indicators of the disease activity - erythrocyte sedimentation rate (ESR) and C-reactive protein (CRP) - as well as prognostic factors like rheumatoid factor (RF), anti-cyclic citrullinated peptide (aCCP) and others, applied in everyday rheumatology practice seem to be insufficient. An analysis of single indicators in laboratory diagnostics for cartilage damage in the course of rheumatoid arthritis or osteoarthritis may not be of much use; however, combining several indicators could make the task easier.

Fibulin-3 (Fib-3), also known as an epidermal growth factor containing a fibulin-like extracellular matrix protein-1 (EFEMP1) and an epidermal growth factor (EGF) containing fibulin-like ECM protein, is an extracellular glycoprotein (one of 7 members of the fibulin family). During development, fibulin-3 is expressed in the mesenchyme, stimulating the growth of bone and cartilage structures. Fibulin-3 stimulates the expression of the tissue inhibitor of metalloproteinase-1 (TIMP-1) and tissue inhibitor of metalloproteinase-3 (TIMP-3), and inhibits the expression matrix metalloproteinases MMP-2, MMP-3 and MMP-9. It also inhibits angiogenesis. ${ }^{2}$ Recently, Wakabayashi et al. showed that the over-expression of fibulin-3 in the clonal murine cell line negatively regulated chondrocyte differentiation. ${ }^{3}$ Compared to age-matched healthy subjects, median levels of serum Fib-3 were elevated in osteoarthritis patients. These findings indicate that Fibulin-3 peptides (Fib3-1 and Fib3-2) may constitute a potential biochemical marker of articular cartilage metabolism. ${ }^{4}$ Fibulin-3 is present in various tissues, including the eye and blood vessels. It is highly expressed in epithelial and endothelial cells and is located in their basement membranes. ${ }^{2}$

The cartilage oligomeric matrix protein (COMP), also designated as thrombospondin 5 (TSP5), is a well-known 425-kDa non-collagenous glycoprotein present in the extracellular matrix of the articular cartilage. COMP was also found in other tissues, including the synovium and tendon. COMP has shown promise as a potential biomarker for monitoring the progression of destruction of the joint cartilage, both in osteoarthritis and in rheumatoid arthritis. ${ }^{5}$

Type II collagen is the basic type of collagen building the articular cartilage; therefore, the urinary $\mathrm{C}$-terminal telopeptide of type II collagen (CTX-II) - indicator of cartilage degradation, as well as procollagen II C-propeptide (PICP) - marker of type II collagen synthesis in the cartilage, may also be helpful in assessing the degree of cartilage damage in arthritis. ${ }^{6}$

MRP8/14 is a myeloid-related protein also known as calprotectin, S100A8/A9 or calgranulin A and B, released from activated granulocytes and macrophages in the synovium and synovial fluid during inflammation. Calprotectin causes proinflammatory effects in vitro on phagocytes and endothelial cells, and promotes inflammation in vivo. ${ }^{7}$ MRP8/14 is a potentially more sensitive biomarker of disease activity in rheumatoid arthritis than conventional inflammatory indexes such as the erythrocyte sedimentation rate (ESR) and C-reactive protein (CRP), because it directly reflects inflammation in the synovium. ${ }^{8}$

The aim of the study was to evaluate the effect of antiTNF therapy on serum fibulin-3, COMP, PIICP, and urinary CTX-II levels in relation to calprotectin and disease activity in rheumatoid arthritis patients.

\section{Patients and methods}

A total of 35 female patients with RA classified according to the revised criteria of the American College of Rheumatology 1987 were qualified for the study. Patient characteristics are shown in Table 1. All the patients had active diseases (Disease Activity Score 28 - DAS28 > 5.1) and did not achieve remission after the application of at least 2 synthetic disease-modifying drugs. Anti-TNF treatment was administered over a 9-month period. Moreover, the study group was divided into patients who responded well to the anti-TNF treatment - called group of responders - comprising patients with remission (DAS28<2.6) and patients with low diseases activity (DAS28 $<3.2$ ), and into those who did not react to the treatment in a sufficient way - group of non-responders (DAS28 > 3.2). Patients were also given methotrexate and prednisone in a stable dose.

Table 1. Patient characteristics

\begin{tabular}{|l|c|}
\hline \multicolumn{1}{|c|}{ RA patients } & Patients $(\mathrm{n}=35)$ \\
\hline Age [years], mean \pm SD & $48.29 \pm 11.55$ \\
\hline Female, $\mathrm{n}(\%)$ & $35(100)$ \\
\hline RA duration [years] & $8.3 \pm 3.1$ \\
\hline DAS28 > 5.1, $\mathrm{n}(\%)$ & $35(100)$ \\
\hline Methotrexate in a dose of 25 mg/week, $\mathrm{n}(\%)$ & $35(100)$ \\
\hline Prednisone (mg/d), mean \pm SD & $2.78 \pm 2.08$ \\
\hline Anti-TNF treatment, $\mathrm{n}(\%)$ & $10(28.5)$ \\
- adalimumab & $5(14.2)$ \\
- certolizumab & $19(54.2)$ \\
- etanercept & $1(3.1)$ \\
\hline
\end{tabular}


The study included only female patients. After a clinical examination and additional test results, it was possible to exclude from this group patients with potential factors which could have had some impact on the concentration of the determined markers, i.e., renal and liver insufficiency, heart failure, endocrinopathy, or neoplastic disease. None of the patients smoked cigarettes. As the control group, 10 healthy women matched in terms of age and body mass index (BMI) were investigated.

The concentration of fibulin-3, COMP, PIICP, MRP8/14, and urinary CTX-II in serum was measured before the treatment as well as following 36 weeks of anti-TNF therapy. Blood and urine samples were taken in the morning (8 a.m.) after overnight fasting. Serum was stored at $-20^{\circ} \mathrm{C}$. The serum fibulin-3 level was measured using an Enzymelinked Immunosorbent Assay Kit (ELISA Kit For Fibulin3 FBLN3; Cloud-Clone Corp., Katy, USA); the concentration of COMP in serum was measured with Human Cartilage Oligomeric Matrix Protein ELISA (BioVendor Research and Diagnostic Products, Laboratorni medicina a.s., Brno, Czech Republic); the serum PIICP level was measured with an Enzyme-linked Immunosorbent Assay Kit (ELISA For Procolagen C-Terminal Propeptide PIICP; Cloud-Clone Corp.); and the urinary CTX-II level was measured with an Enzyme-linked Immunosorbent Assay Kit (ELISA For Cross Linked C Telopeptide Of Type II Collagen - CTX-II; Cloud-Clone Corp.); urinary CTX-II level was corrected by the urinary creatinine $(\mathrm{Cr})$ concentration. No patients had marked kidney or liver function impairment that may have altered the urinary CTX-II levels.

The concentration of MRP8/14 in serum was measured with a PhiCal ${ }^{\circledR}$ Calprotectin ELISA Kit (Immundiagnostik, Bensheim, Germany).
The erythrocyte sedimentation rate and C-reactive protein level were assayed with routinely used methods. A total of 28 joints (proximal inter-phalangeals 1-5, metacarpophalangeals 1-5, wrists, elbows, shoulders, and knees) were assessed with regard to swelling and pain. DAS28 was calculated as the disease activity index. Hemoglobin concentration was taken into account in the study group. According to recent findings, it may be closely related to the disease activity and progression of radiological changes in patients with RA. ${ }^{9}$

\section{Statistical analysis}

The obtained results were expressed as mean \pm standard deviation (SD). Significant difference was determined by adopting a limiting error value equal to $95 \%$, which corresponds to $\mathrm{p}<0.05$. Calculations for dependent samples were carried out using the Wilcoxon non-parametric pair test. The Mann-Whitney U test was used to carry out comparisons between the groups. An analysis of correlations was conducted using Spearman's R rank correlation. Meanwhile, multiple regression analysis was applied in order to determine the existing interdependences.

\section{Results}

Table 2 presents the concentration of fibulin-3, COMP, uCTX-II, PIICP, and MRP8/14, values of ESR, CRP, DAS28, the number of painful and swollen joints, and the concentration of hemoglobin in the serum of RA patients before and after the treatment, divided into responders (63\%) and non-responders (37\%), as well as the mean values of the

Table 2. The results (mean \pm SD) regarding fibulin-3, COMP, UCTX-II, PIICP, MRP8/14, ESR, CRP, DAS28, TEN, SW, and HGB before the treatment (baseline) and after 9 months of anti-TNF therapy

\begin{tabular}{|c|c|c|c|c|c|c|c|}
\hline \multirow[t]{2}{*}{ Markers } & \multicolumn{2}{|c|}{$\begin{array}{l}\text { All patients } \\
\quad(n=35)\end{array}$} & \multicolumn{2}{|c|}{$\begin{array}{l}\text { Responders } \\
\qquad(\mathrm{n}=22)\end{array}$} & \multicolumn{2}{|c|}{$\begin{array}{l}\text { Non-responders } \\
\quad(n=13)\end{array}$} & \multirow{2}{*}{$\begin{array}{l}\text { Control group } \\
\qquad(n=10)\end{array}$} \\
\hline & baseline & after therapy & baseline & after therapy & baseline & after therapy & \\
\hline Fibulin-3 [ng/mL] & $110.25 \pm 50.86$ & $77.03 \pm 48.98^{*}$ & $98.32 \pm 48.16$ & $64.73 \pm 44.31^{*}$ & $130 \pm 5.68$ & $97.85 \pm 51.68^{*}$ & $83 \pm 23.52^{* * *}$ \\
\hline COMP [ng/mL] & $1189.13 \pm 399.21$ & $1088.43 \pm 28.55$ & $1159.40 \pm 374.63$ & $1126.16 \pm 293.86$ & $1239.44 \pm 448.98$ & $1024.57 \pm 257.78$ & $1122.71 \pm 243.31$ \\
\hline uCTX-II [pg/mL] & $438.14 \pm 244.43$ & $406.14 \pm 267.50$ & $402.79 \pm 269.40$ & $486.62 \pm 273.26$ & $497.96 \pm 189.99$ & $269.95 \pm 200.25^{*}$ & $414.17 \pm 154.99$ \\
\hline PIICP [pg/mL] & $2767.20 \pm 953.66$ & $3232.59 \pm 1326.95$ & $2814.11 \pm 961.16$ & $3554.88 \pm 1477.09$ & $2687.82 \pm 974.30$ & $2687.17 \pm 811.24$ & $3481.68 \pm 1186.0$ \\
\hline MRP8/14 [ng/mL] & $2421.83 \pm 2015.28$ & $844.35 \pm 702.93^{*}$ & $2465.93 \pm 2228.63$ & $733.31 \pm 723.15^{*}$ & $2347.22 \pm 1675.02$ & $1032.26 \pm 65^{*}$ & $404.97 \pm 211.58^{* *}$ \\
\hline $\mathrm{ESR}[\mathrm{mm} / \mathrm{h}]$ & $35.08 \pm 23.91$ & $18.34 \pm 12.45^{*}$ & $30.72 \pm 20.37$ & $15.77 \pm 11.15^{*}$ & $42.46 \pm 28.30$ & $22.69 \pm 13.75^{*}$ & - \\
\hline CRP $[\mathrm{mg} / \mathrm{L}]$ & $15.09 \pm 21.07$ & $6.04 \pm 6.46^{*}$ & $10.91 \pm 11.04$ & $5.05 \pm 4.74^{*}$ & $22.16 \pm 30.95$ & $7.715 \pm 8.62$ & - \\
\hline DAS 28 & $6.65 \pm 0.91$ & $2.95 \pm 1.07^{*}$ & $6.47 \pm 0.91$ & $2.37 \pm 0.61^{*}$ & $6.97 \pm 0.86$ & $3.93 \pm 97^{*}$ & - \\
\hline TEN & $18.27 \pm 7.64$ & $2.11 \pm 2.59^{*}$ & $17.86 \pm 8.10$ & $1.09 \pm 1.30^{*}$ & $18.92 \pm 7.07$ & $3.84 \pm 3.31^{*}$ & - \\
\hline SW & $12 \pm 7.65$ & $1.28 \pm 2.81^{*}$ & $10.81 \pm 7.76$ & $0.36 \pm 0.72^{*}$ & $14 \pm 7.31$ & $2.84 \pm 4.16^{*}$ & - \\
\hline HGB [g\%] & $12.90 \pm 1.5$ & $13.44 \pm 1.22^{*}$ & $12.9 \pm 1.37$ & $13.63 \pm 1.16^{*}$ & $12.91 \pm 1.75$ & $13.12 \pm 1.30$ & - \\
\hline
\end{tabular}

COMP - cartilage oligomeric matrix protein; UCTX-II - C-terminal telopeptide of collagen type II; PIICP - C-propeptide of collagen type II; MRP8/14 - calprotectin; ESR - erythrocyte sedimentation rate; CRP - C-reactive protein; DAS28 - diseases activity score 28; TEN - tender joint count; SW - swollen joint count; HGB - hemoglobin concentration; * statistical significance of the difference (baseline vs after therapy) $p$-value $<0.05$; ${ }^{* *}$ statistical significance of the difference (all patients before treatment (baseline) vs control group), p-value $<0.05 ;{ }^{* * *}$ statistical significance of the difference (non-responders before treatment (baseline) vs control group), p-value $<0.05$. 
abovementioned parameters (with the exception of ESR, CRP, DAS28, the number of painful and swollen joints, and the hemoglobin concentration) measured in the control group.

The mean serum concentration of fibulin-3 in RA patients before the treatment did not differ significantly from the mean serum concentration of fibulin-3 in the blood serum of women from the control group.

Statistically significant higher mean concentration of fibulin-3 compared to the mean concentration of fibulin-3 in the serum of healthy women was noted prior to treatment in the group of women with a worse response to the anti-TNF therapy (non-responders).

After 9 months of treatment, a statistically significant decrease in the mean concentration of fibulin-3 was observed in the serum of all patients with rheumatoid arthritis, treated with TNF inhibitors, both in the group of responders and in the group of non-responders.

No statistically significant difference was noted between the mean concentration of COMP in the serum of patients from the study group before the treatment (including responders and non-responders) and the mean concentration of COMP in the serum of healthy women. After 9 months of anti-TNF therapy, no significant changes were noted in the mean concentration of COMP in the serum of RA patients, although the overall trend showed a decrease in the concentration of COMP after the treatment (Table 2).

No statistically significant difference was noted between the mean concentration of PIICP in the serum of patients from the study group before the treatment (including responders and non-responders) and the mean concentration of PIICP in the serum of healthy women. After treatment, no statistically significant differences were observed in the mean concentration of PIICP in the serum of RA patients. However, the overall trend showed an increasing concentration of PIICP after the treatment (Table 2).
The mean uCTX-II concentration in patients constituting the control group did not differ from the mean uCTX-II concentration in women from the control group. The uCTX-II concentration in RA patients did not change significantly following the treatment. Only after taking into account the responders and non-responders in the study group was it stated that a statistically significant decrease of UCTX-II concentration in urine for women treated with TNF inhibitors was observed in the group that did not respond to therapy properly.

A statistically significant higher mean concentration of MRP 8/14 was noted in the serum of RA patients before the treatment compared to the mean MRP8/14 concentration in the serum of healthy women. After 9 months of observation, a significant decrease of the mean concentration of MRP8/14 was noted in the serum of patients after anti-TNF therapy, both in the group which achieved its therapeutic goal (low disease activity or remission) and in those patients who did not experience a decrease of the DAS28 value $<3.2$.

The ESR, CRP level, the DAS28 index, as well as the number of painful and swollen joints were decreased in all patients following the treatment. A decrease in CRP concentration in serum was not statistically significant in the group of patients with insufficient response to therapy. Following anti-TNF therapy, the concentration of hemoglobin increased significantly in all patients as well as in the group of patients who positively responded to the treatment. Such a tendency was not observed in the group of non-responders.

Correlations between fibulin-3 and the remaining parameters in the study groups are presented in Table 3.

In the group of RA patients, a statistically significant correlation was observed between the concentrations of fibulin-3 and COMP in the serum before the treatment. A positive significant correlation was also noted

Table 3. Correlation between serum fibulin-3 level and other markers in RA patients before and after anti-TNF therapy

\begin{tabular}{|l|c|c|c|c|c|c|}
\hline \multirow{2}{*}{ Correlation } & \multicolumn{2}{|c|}{ All patients } & \multicolumn{2}{c|}{ Responders } & \multicolumn{3}{c|}{ Non-responders } \\
\cline { 2 - 7 } & baseline & after therapy & baseline & after therapy & baseline & after therapy \\
\hline Fib-3/COMP & $R$ & $R$ & $R$ & $R$ & $R$ \\
\hline Fib-3/UCTX-II & $0.404^{*}$ & 0.299 & 0.376 & $0.534^{*}$ & 0.461 & 0.060 \\
\hline Fib-3/PIICP & 0.104 & -0.314 & 0.124 & -0.285 & -0.351 & 0.236 \\
\hline Fib-3/MRP8/14 & -0.242 & -0.097 & -0.221 & 0.088 & -0.439 & -0.027 \\
\hline Fib-3/ESR & 0.380 & 0.269 & 0.373 & 0.027 & 0.478 & -0.076 \\
\hline Fib-3/CRP & 0.333 & $0.367^{*}$ & 0.392 & 0.171 & 0.208 & 0.544 \\
\hline Fib-3/DAS 28 & 0.255 & $0.411^{*}$ & 0.311 & $0.455^{*}$ & 0.256 & 0.345 \\
\hline Fib-3/TEN & $0.351^{*}$ & 0.310 & 0.244 & -0.123 & 0.412 & 0.398 \\
\hline Fib-3/SW & 0.043 & 0.096 & -0.041 & -0.300 & 0.218 & 0.207 \\
\hline Fib-3/HGB & 0.267 & 0.189 & 0.371 & 0.262 & 0.179 & -0.313 \\
\hline
\end{tabular}

Fib-3 - serum fibulin-3; COMP - cartilage oligomeric matrix protein; UCTX-II - C-terminal telopeptide of collagen type II; PIICP - C-propeptide of collagen type II; MRP8/14 - calprotectin; ESR - erythrocyte sedimentation rate; CRP - C-reactive protein; DAS28 - diseases activity score 28; TEN - tender joint count; SW - swollen joint count; HGB - hemoglobin concentration; ${ }^{*}$ statistically significant $p$-value $<0.05$. 
between the concentrations of fibulin-3 and DAS28 before the treatment. Following anti-TNF therapy, a significant positive correlation was observed between the concentrations of fibulin-3 and selected inflammatory indexes (ESR and $(R P)$ in the entire group of patients. After the treatment, a significant positive correlation between fibulin-3 and CRP concentrations was observed also in the group of responders. Moreover, in this group there was a significant correlation between fibulin-3 and COMP concentrations. In the study groups, no other significant relations were stated between fibulin-3 and the remaining tested indicators of cartilage metabolism, inflammatory indexes, painful and swollen joints, and hemoglobin concentration.

In the group of healthy women who constituted the control group, no statistically significant correlations were noted between fibulin-3 and COMP, uCTX-II, PIICP, and MRP8/14.

In the group of RA patients, relations between the remaining cartilage metabolism markers (uCTX-II, PIICP, COMP) and inflammatory indexes were small. A significant negative correlation was shown in the entire group of patients between PIICP and CRP concentrations $(R=-0.505$; $\mathrm{p}<0.005)$ before the treatment, and between UCTX-II and DAS28 $(R=-0.348 ; p<0.05)$ after the treatment. In the group of non-responders, a correlation was noted between the COMP concentration and SW $(\mathrm{R}=0.589$; $\mathrm{p}<0.005)$ following the treatment. Moreover, a correlation between PIICP and hemoglobin concentrations $(\mathrm{R}=0.752$; $\mathrm{p}<0.005)$ was stated in this group before the treatment.

Correlations between MRP8/14 and the remaining parameters in the RA patients' groups are presented in Table 4. A statistically significant positive correlation between MRP8/14 and ESR and CRP was observed before the treatment in the entire group of RA patients and in the group of responders. However, such a relation did not occur in those groups after the treatment, nor did it appear in the group of non-responders. In the group of responders, a significant correlation after the treatment was reported only between MRP8/14 and the number of painful joints. In the group of non-responders, a significant negative correlation between MRP8/14 and hemoglobin concentrations was observed before the treatment. In none of the tested groups of RA patients were there statistically significant relations observed between the serum MRP8/14 concentration and the serum COMP and PIICP concentrations and UCTX-II.

In the control group, a significant negative correlation was noted between the serum MRP8/14 level and UCTX-II ( $\mathrm{R}=0.709 ; \mathrm{p}<0.05)$.

The conducted multivariate regression analysis with a dependent variable fibulin-3, and independent variables such as the concentration of COMP, uCTX-II, PIICP, MRP8/14, ESR, CRP, TEN, SW and hemoglobin concentration in RA patients after 9 months of anti-TNF therapy, has shown a significant positive correlation between the concentration of fibulin-3 in serum and the concentration of CRP $(\beta=0.550 ; p=0.000)$.

The multivariate regression analysis (multi-factor analysis) with a dependent variable MRP8/14, and independent variables - cartilage metabolism markers - such as the concentration of COMP, uCTX-II, PIICP, and fibulin-3 in RA patients after 9 months of anti-TNF therapy, has shown a significant negative correlation only between the concentration of PIICP in serum and the concentration of serum MRP8/14 ( $\beta=-0.408 ; \mathrm{p}<0.05)$.

\section{Discussion}

One of the more important and interesting discoveries in recent years involved the identification of fibulin in the serum of osteoarthritis patients. Henrotin et al. suggest that fibulin-3 peptides (Fib 3-1 and Fib 3-2) are potential biomarkers of osteoarthritis. ${ }^{4}$ The search for reports on fibulin-3 in patients with rheumatoid arthritis

Table 4. Correlation between serum MRP8/14 level and others markers in RA patients before and after anti-TNF therapy

\begin{tabular}{|c|c|c|c|c|c|c|}
\hline \multirow{3}{*}{ Correlation } & \multicolumn{2}{|c|}{ All patients } & \multicolumn{2}{|c|}{ Responders } & \multicolumn{2}{|c|}{ Nonresponders } \\
\hline & baseline & after therapy & baseline & after therapy & baseline & after therapy \\
\hline & $\mathrm{R}$ & $\mathrm{R}$ & $\mathrm{R}$ & $\mathrm{R}$ & $\mathrm{R}$ & $\mathrm{R}$ \\
\hline MRP8/14/COMP & 0.030 & 0.111 & 0.136 & 0.031 & -0.208 & -0.016 \\
\hline MRP8/14/uCTX-II & 0.009 & -0.282 & -0.000 & -0.177 & -0.060 & -0.483 \\
\hline MRP8/14/PIICP & -0.535 & -0.569 & -0.457 & -0.360 & -0.752 & -0.769 \\
\hline MRP8/14/ESR & $0.533^{*}$ & 0.268 & $0.544^{*}$ & 0.193 & 0.445 & 0.272 \\
\hline MRP8/14/CRP & $0.525^{*}$ & 0.271 & $0.521^{*}$ & 0.181 & 0.457 & 0.339 \\
\hline MRP8/14/DAS 28 & 0.182 & 0.142 & 0.207 & -0.233 & 0.225 & 0.035 \\
\hline MRP8/14/TEN & -0.072 & -0.018 & 0.009 & $0.441^{*}$ & -0.033 & 0.125 \\
\hline MRP8/14/SW & 0.052 & 0.004 & 0.274 & -0.210 & -0.382 & -0.135 \\
\hline MRP8/14/HGB & -0.295 & -0.090 & -0.097 & 0.151 & $-0.648^{*}$ & -0.465 \\
\hline
\end{tabular}

MRP8/14 - calprotectin; COMP - cartilage oligomeric matrix protein; UCTX-II - C-terminal telopeptide of collagen type II; PIICP - C-propeptide of collagen type II; ESR - erythrocyte sedimentation rate; CRP - C-reactive protein; DAS28 - diseases activity score 28; TEN - tender joint count; SW - swollen joint count; HGB - hemoglobin concentration; * statistically significant $p$-value $<0.05$. 
in the Medline database returned no results. In rheumatoid arthritis, just like in osteoarthritis, articular cartilage is damaged. Also, the pathomechanism of this phenomenon seems to be different. Advanced destructive changes may be confirmed by imaging tests (MRI, USG or X-ray), but so far no biochemical marker or group of biomarkers have been found. That would probably allow for a quicker and more precise monitoring of cartilage damage, and help with the identification of groups of patients more or less responsive to modern treatment, thus enabling quicker therapy verification.

The obtained results indicate a lack of differences in the concentration of fibulin-3 in serum between RA patients and healthy individuals (only in the group of non-responders was the concentration of fibulin-3 before the treatment higher than in the control group).

In RA patients treated with anti-TNF inhibitors, a statistically significant decrease of fibulin-3 concentration in serum was observed after the treatment. Interestingly, the concentration of fibulin-3 was decreased both in the group of responders and in the group of non-responders. Lack of literature data regarding this issue makes it impossible to compare the obtained results with those of other authors. Increased concentration of fibulin-3 peptides in serum (Fib3-1 and Fib3-2) was described only in osteoathritis patients. ${ }^{4}$ The results of this work suggest that TNF blockade modifies the release and expression of fibulin-3 in RA patients. This is also illustrated by the fact that after the treatment, and therefore after lowering the degree of inflammation, the concentration of fibulin-3 in serum strictly correlated with the value of ESR and CRP in the entire group of RA patients (in the group of responders - correlation only with CRP concentration). Such a relation was not observed between fibulin-3 and MRP8/14, although Garcia-Arias et al. stated that serum MRP8/14 levels strongly correlated with laboratory assessments of joint inflammation in RA patients (ESR and CRP). ${ }^{8}$

With regard to the relation between fibulin-3 and other biomarkers of cartilage metabolism assessed in this study, the concentration of fibulin-3 in the group of RA patients did not correlate (both before and after the treatment) with the concentration of urinary CTX-II and the concentration of PIICP in serum. Contrary to the observations of Marotte et al., the concentrations of UCTX-II and PICP did not significantly differ between the tested group before the treatment and the control group. ${ }^{10}$ Moreover, these concentrations did not change in a statistically significant way following 9-month anti-TNF therapy (except for the significant decrease of uCTX-II concentration in the group of non-responders). Meanwhile, the lack of changes in UCTX-II concentration for RA patients during the treatment coincides with the observations of Marotte et al., conducted during a 1-year observation of patients treated with infliximab. ${ }^{10}$ The concentration of COMP in the serum of RA patients did not change after anti-TNF therapy as well. On the other hand, Crnkic M. et al. described in their report the decrease of COMP concentration in the serum of RA patients treated with infliximab or etanercept. ${ }^{11}$

Meanwhile, in this work a statistically significant correlation was observed between the concentrations of fibulin-3 and COMP in RA patients before the treatment and after the treatment in the group of responders.

The concentration of MRP8/14 was higher in the group of RA patients before the treatment than in the control group, and it decreased in a statistically significant way in all groups of RA patients after anti-TNF therapy, which is in accordance with the results obtained by other authors. ${ }^{8}$ As expected, after the treatment, the values of ESR, CRP, DAS 28, TEN, and SW decreased, but not all patients achieved remission or low disease activity.

After the treatment, a significant increase was noted in hemoglobin concentration in all groups of RA patients (also in the group of responders and non-responders). However, its concentration did not correlate with the concentration of fibulin-3 before or after the treatment.

It seems that further studies are needed in order to gain a better understanding of the role of fibulin-3 in the cartilage metabolism of RA patients.

The correlation the cartilage biomarkers with other inflammatory factors such as tumor necrosis factor, interleukin- 6 and interleukin-17 seems to be a very interesting subject for further investigations.

\section{Conclusions}

Higher concentration of fibulin-3 in the serum was found in RA patients who did not respond to the treatment in a sufficient way (non-responders).

During the anti-TNF therapy, the serum fibulin-3 level decreased in patients regardless of whether they achieved remission or low disease activity.

The concentration of fibulin-3 correlated with CRP and ESR after the treatment in the entire group of patients.

No correlation between fibulin-3 and MRP8/14 was observed in the study group (before and after the treatment) nor in the control group.

A correlation between fibulin-3 and COMP in the group of RA patients before and after the treatment was observed only in the group of responders.

The analysis of multivariate regression indicated that the concentration of $\mathrm{C}$-reactive protein in serum is the most significant parameter (assessed in this work) with which the concentration of fibulin-3 in serum correlates.

\section{References}

1. Rousseau JC, Garnero P. Biological markers in osteoarthritis. Bone. 2012;51:265-277.

2. Zhang Y, Marmorstein LY. Focus on molecules: Fibulin-3 (EFEMP1). Exp Eye Res. 2010;90:374-375.

3. Wakabayashi T, Matsumine A, Nakazora S, et al. Fibulin-3 negatively regulates chondrocyte differentiation. Biochem Biophys Res Commun. 2010;391:1116-1121. 
4. Henrotin Y, Gharbi M, Mazzucchelli G, Dubuc JE, De Pauw E, Deberg M. Fibulin 3 peptides Fib3-1 and Fib3-2 are potential biomarkers of osteoarthritis. Arthritis Rheum. 2012;64:2260-2267.

5. Larrson E, Erlandsson Harris H, Lorentzen JC, et al. Serum concentrations of cartilage oligomeric matrix protein, fibrinogen and hyaluronan distinguish inflammation and cartilage destruction in experimental arthritis in rats. Rheumatology. 2002;41:996-1000.

6. Elsaid KA, Chichester CO. Review: Collagen markers in early arthritic diseases. Clin Chim Acta. 2006;365:68-77.

7. Brun JG, Jonsson R, Haga HJ. Measurement of plasma calprotectin as an indicator of arthritis and diseases activity in patients with inflammatory rheumatic disaeses. J Rheumatol. 1994;21:733-738.

8. García-Arias M, Pascual-Salcedo D, Ramiro S, et al. Calprotectin in rheumatoid arthritis. Mol Diagn Ther. 2013;17:49-56.
9. Möller B, Scherer A, Förger F, Viliger P, Finckh A. Anaemia may add information to standardized disease activity assessment to predict radiographic damage in rheumatoid arthritis: A prospective cohort study. Ann Rheum Dis. 2014;73:691-696.

10. Marotte H, Gineyts $E$, Miossec P, Delmas PD. Effects of infliximab therapy on biological markers of synovium activity and cartilage breakdown in patients with rheumatoid arthritis. Ann Rheum Dis. 2009;68:1197-1200.

11. Crnkic M, Mansson B, Larsson L, Geborek P, Heinegard D, Saxne T. Serum cartilage oligomeric matrix protein (COMP) decreases in rheumatoid arthritis patients treated with infliximab or etanercept. Arthritis Res Ther. 2003;5:181-185. 